\title{
Do hyperspectral transmissometers allow us to go one step further on the analysis of particulate matter characteristics of marine samples?
}

\author{
Ramírez-Pérez M., Torrecilla E., Sánchez A.M., Piera J. \\ Institute of Marine Sciences \\ Spanish National Research Council (ICM-CSIC) \\ Barcelona, Spain \\ mramirez@icm.csic.es
}

\begin{abstract}
Several studies have been carried out to investigate the correlation between the spectral shape features of the beam attenuation coefficient and the particulate matter characteristics in seawater, but little attention has been paid to the spectral resolution of these measurements. For this reason, the potential of the new hyperspectral transmissometer VIPER (TriOS $\mathrm{GmbH}$ ), with $1.7 \mathrm{~nm}$ spectral resolution, has been evaluated in this study and compared with lower resolution and multispectral based approaches (e.g. ac-9 or ac-s -with $4 \mathrm{~nm}$ resolution- from WETLabs Inc.) in order to evaluate whether any additional information about water composition can be retrieved from a spectral shape-based assessment. In this way, this study proposes a statistical-based method -a Hierarchical Cluster Analysis (HCA) using the cosine distance as similarity value- which allows discriminating suspended sediment samples with different particle size distribution (PSD) based on the attenuation spectral shape features. Finally, the effects of both particle size and concentration on the spectral shape have been analyzed separately. The results confirmed that the beam attenuation spectral features are in first-order driven by particle concentration, which means that a prior knowledge of particulate matter concentration is required in order to classify sediment samples according to their particle size. This approach based on hyperspectral attenuation measurements to characterize the PSD has been demonstrated a potential alternative compared to the traditional methods such as Coulter Counter or the particle size analyzer LISST 100X, which are much more expensive and timeconsuming approaches.
\end{abstract}

Keywords-hyperspectral transmissometer, beam attenuation spectrum, particle size distribution and concentration, hierarchical cluster analysis.

\section{INTRODUCTION}

For many research fields, there is much interest in characterizing not only the concentration but also the size distribution of the suspended particulate matter in coastal waters, due to their importance in, for example, suspended sediment transport models -the particle size controls the particle settling velocity- and water transparency analysis- it influences the penetration of sunlight into the sea with consequent implications for primary productivity [1-3]. For this reason, and due to the limitations of the traditional instruments (e.g., Coulter Ccounter or imaging techniques), various optical proxies have been used to overcome this challenge [3]. In particular, the beam attenuation has been widely used due to a large number of advantages of this optical property. On one hand, it's almost a direct measurement and non-corrections are required (such as, non-scattering corrections) Furthermore, transmissometers are inexpensive and easy-to-handle instruments. On the other hand, it is well known that the magnitude of the beam attenuation is directly correlated with the total suspended matter (TSM) concentration and its slope covaries with the Particle Size Distribution (PSD). In this sense, it has been proven valid as a proxy of the water compounds properties $[1,4,5,6]$.

The transmissometers are characterized by their path length, the acceptance angle (or field of view, FOV), the covered spectral range and the spectral resolution. Most of the studies related to beam attenuation for the analysis of particulate matter characteristics carried out up to now, have been based on either multispectral sensors (e.g. WET Labs ac9) or the hyperspectral spectrophotometer WET Labs ac-s with a spectral resolution of $4 \mathrm{~nm}[1,4,6,7,8,9]$. However, a new hyperspectral transmissometer (i.e., VIPER, from TriOS $\mathrm{GmbH}$ ) has recently been designed with a spectral resolution of $1.7 \mathrm{~nm}$, which provides the opportunity to assess whether there is any additional shape feature on the beam attenuation spectrum which allows discriminating between different particulate matter characteristics (i.e., particle size and concentration) and improving our capability to retrieve more information about water composition, similarly to other past studies $[10,11,12]$. For instance, Torrecilla et al. [12] classified different water masses and phytoplankton pigment assemblages from the Eastern Atlantic Ocean, according to the similarity of hyperspectral observations of remote sensing reflectances. In this case, an unsupervised Hierarchical Cluster Analysis (HCA) was used based on a cosine distance as a measure of similarity between each pair of spectra.

In this study, in order to explore the capability to discriminate samples with different particulate matter characteristics, the HCA similarity analysis has been applied to a dataset of beam attenuation observations measured in the laboratory using the VIPER hyperspectral instrument.

\section{METHODOLOGY}

\section{A. Experimental set-up:}

Sediment samples were collected in the North Western Mediterranean coast and classified into different grain size ranges using sieves. Six different particle sizes ranges, from 
mud $(<5 \mu \mathrm{m})$ to fine sand $(125-250 \mu \mathrm{m})$ were obtained. These samples were diluted into glycerol at different concentrations $(50,100,200,500$ and $1000 \mathrm{mg} / \mathrm{L})$ and carefully stirred by hand in order to obtain a homogeneous distribution and avoid air bubbles generation. Due to the difficulties to achieve a homogeneous particle concentration in a recirculation tank, glycerol was used as solution instead of water. The higher viscosity of glycerol allowed the sediment particles to be kept in homogeneous suspension for longer periods of time without the need for re-circulation [13].

The transmission spectrum was measured several times for each prepared sample using the VIPER hyperspectral sensor (TriOS GmbH), during 10 minutes and a 30 seconds sampling period. Furthermore, each test was repeated five times, leading to a dataset of 100 spectra for each sample (i.e., each combination of particle grain size and concentration). Each transmission spectrum was measured with the VIPER within the spectral range from 350 to $750 \mathrm{~nm}$ with a $1.7 \mathrm{~nm}$ resolution (i.e., a total of 255 wavelengths). Given that the instrument pathlength is $5 \mathrm{~cm}$, its application is suitable for very turbid water environments (e.g. surf zone, river plumes) and permitted to carry out the experiment based on the abovementioned very high suspended sediment concentrations. The instrument FOV is $<1^{\mathrm{O}}$ (in process to be exactly determined by the manufacturer).

Based on the measured transmission signal, the beam attenuation spectrum was determined by applying the following equation:

$$
c(\lambda)=-(1 / r) \cdot \operatorname{Ln} T(\lambda)
$$

where $r$ is the instrument path length $(0.05 \mathrm{~m}$ in this case).

In order to have additional information on particle concentration and size distribution of each considered sample, simultaneous measurements were performed with the LISST100X Type B instrument (Laser In Situ Scattering and Transmissometry, from Sequoia Scientific, Inc.). This instrument estimates the PSD in 32 logarithmically spaced size classes ranged from 1.25 to $250 \mu \mathrm{m}$ based on laser beam diffraction. LISST data processing was carried out using the Matlab scripts provided by the manufacturer and each particle size and volume concentration from the raw near-forward scattering data were obtained. Furthermore, the particle number concentration for each size bin was estimated by dividing the obtained volume concentration by the volume of the equivalent sphere [14].

\section{B. Statistical analysis}

With the aim to classify the measured spectra according to the relative similarity of their spectral shape, a HCA was applied using the beam attenuation spectra as input vectors (or objects). HCA separates a given collection of unlabeled input data into clusters or groups of objects $[12,15]$, based on a pairwise distance (cosine distance, in this case) computed between all objects included in the dataset. The cosine distance, $\mathrm{d}$, is calculated as one minus the cosine of the angle $\theta$ between each pair of objects.

The results were represented in a MDS graphic, which is an ordination technique that, similar to cluster techniques, ranks objects according to their relative similarity [16]. MDS is based on the dissimilarity matrix obtained in the HCA analysis and displays the interrelations between each pair of spectra on a low dimensional plot (2D). The stress value given for an MDS ordination indicates the usefulness of the output. The lower the stress value, the more accurate the output in describing the data set [16].

\section{RESULTS AND DISCUSSION}

\section{A. Discrimination of sediment samples with different PSD based on hyperspectral attenuation measurements}

Fig. 1 shows high spectral resolution measurements of different sediment samples gathered with the VIPER transmissometer. Different singularities on the beam attenuation spectral shape associated with the PSD, such as the slope between $420-450 \mathrm{~nm}$ and $440-480 \mathrm{~nm}$ or the area between 400-425 $\mathrm{nm}$ and 480-520 $\mathrm{nm}$ can be identified from these measurements. In Fig. 2 these differences can be clearly observed on the results obtained from the application of HCA to this dataset. The spectra with different PSD are successfully discriminated as represented in the MDS graphic.

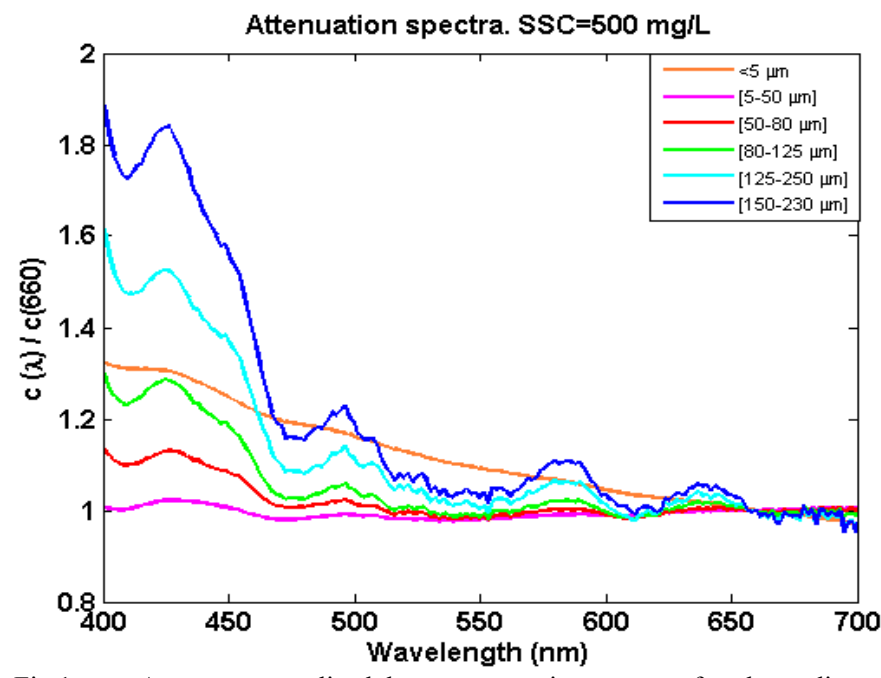

Fig 1. Average normalized beam attenuation spectra for the sediment samples at a constant mass concentration of $500 \mathrm{mg} / \mathrm{L}$ and different grain sizes.

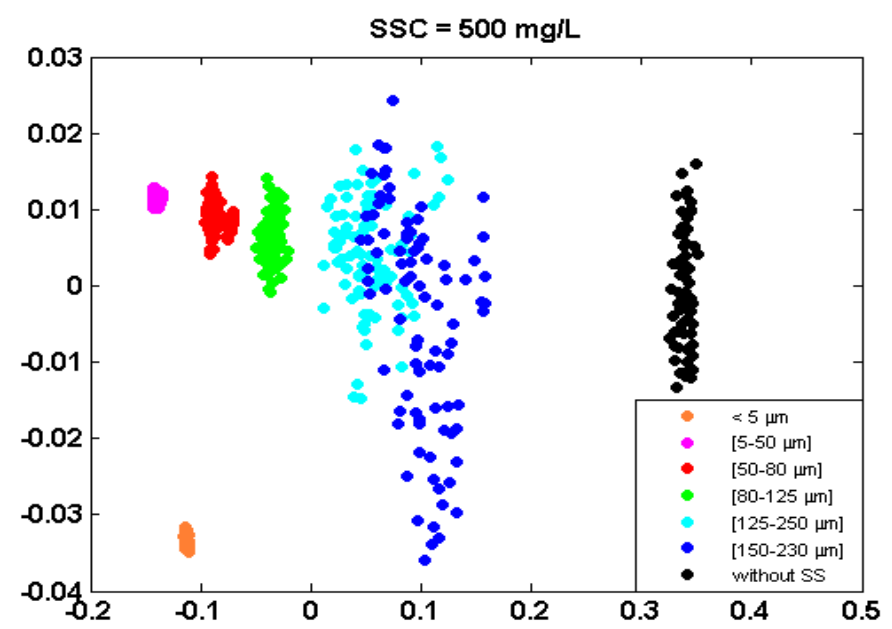

Fig 2. MDS graphic with the classification of the different spectra shown in the Fig. 1 according to their shape similarities. 
This methodology based on the analysis of the whole spectrum features (i.e.. not only on the spectral slope or specific spectral ratios, as has commonly been performed), has been demonstrated a powerful approach for classifying spectra corresponding to different sediment samples according to their PSD. Furthermore, a correlation between the location of the spectra on the MDS graphic and the transparency of the sample has been found. For the same mass concentration, the transparency increases when increasing the particle size, associated with lower particle concentration. Hence, the transparency of our sediment samples increases from left to right side of the MDS plot.

Fig. 3 and Fig. 4 show how the above-mentioned discrimination has also been obtained emulating lower spectral resolution transmissometers. This figure shows the MDS results obtained after applying the HCA to a subset of beam attenuation spectra using only the wavelengths available in the ac-9 and ac-s sensors (left and right panels, respectively).

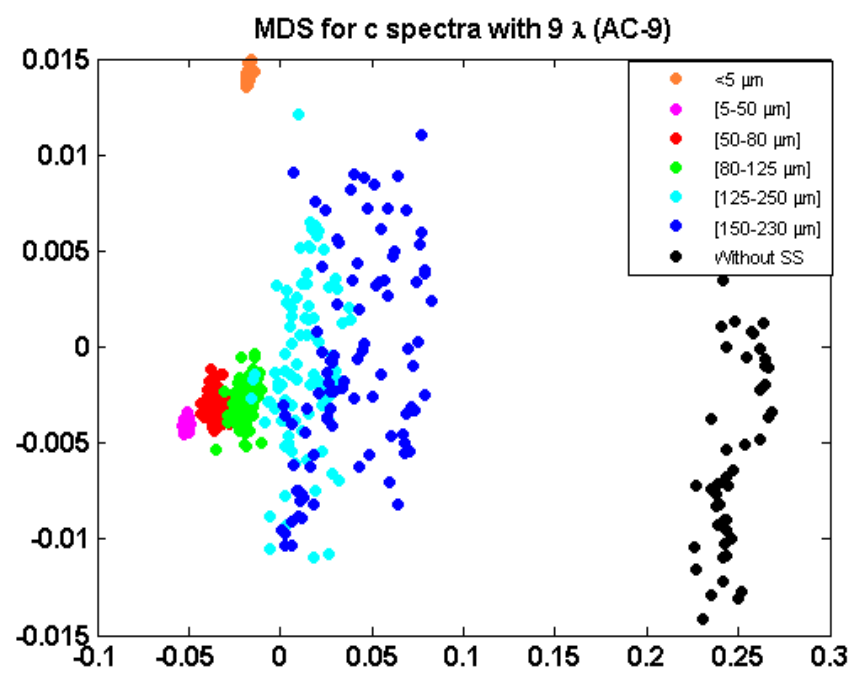

Fig 3. The same as Fig. 2 but when considering attenuation measurements collected at just 9 wavelengths.

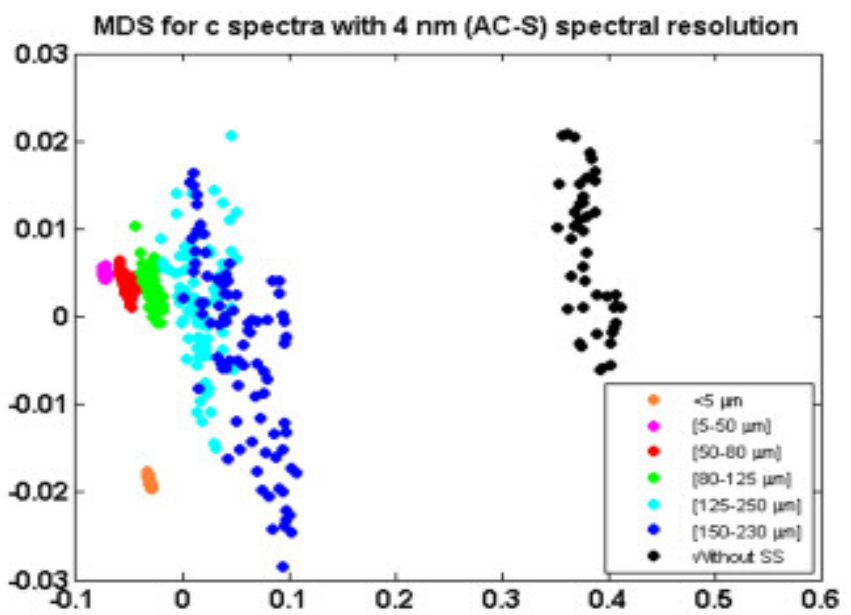

Fig 4. The same as Fig. 2 but when considering attenuation measurements collected at 82 wavelengths.
The differences between each sediment sample, however, are not as significant as those obtained with the VIPER hyperspectral resolution. In fact, the capability of distinguishing samples with different sediment characteristics improves when increasing the spectral resolution (see the different scales of the MDS graphic). These differences could play a key role when analyzing data measured in natural environments, since water composition is more complex and several optically active compounds are present.

\section{B. Influence of particle size and concentration on the beam attenuation spectral shape.}

As described, previous classification discriminated spectra with different particle size distributions (i.e. different particle concentration as a function of size [17]). It was achieved after considering several sediment samples with the same mass concentration $(\mathrm{mg} / \mathrm{L})$, different particle sizes and therefore, different particle concentration as validated by the LISST100X instrument (see Table 1, fourth column). If this analysis had been performed regardless the mass concentration, no clear distinction would have been obtained (results not shown), due to the fact that not only the magnitude but also the spectral shape features of the beam attenuation are highly influenced by the particle concentration $[1,2,8]$. As shown in Fig. 5 and Fig. 6 , this fact can be observed when analyzing a single sediment sample with a grain size $<5 \mu \mathrm{m}$ but at different mass concentrations. In this case, variations on the spectral shape are a consequence of the particle concentration.

TABLE 1. SUMMARY OF RESUlTS OBTAINED FORM LISST 100-X MEASUREMENTS FOR ALL SEDIMENT SAMPLES.

\begin{tabular}{|c|c|c|c|c|}
\hline $\begin{array}{l}\text { Grain size } \\
\quad(\mu \mathrm{m})\end{array}$ & $\begin{array}{c}\text { Mass conc. } \\
(\mathrm{mg} / \mathrm{l})\end{array}$ & $\begin{array}{c}\text { Total } \\
\text { Volume } \\
\text { Conc. } \\
(\mu \mathrm{l} / \mathrm{l})\end{array}$ & $\begin{array}{c}\text { Total } \\
\text { particle } \\
\text { conc. } \\
\left(\operatorname{part}^{\circ} \cdot \boldsymbol{\mu m}^{-}\right. \\
\left.{ }^{1} \cdot \mathbf{L}^{-1}\right)\end{array}$ & $\begin{array}{c}\text { Median } \\
\text { size } \\
\text { diameter, } \\
\mathbf{d}_{50}(\mu \mathrm{m})\end{array}$ \\
\hline$[5-50]$ & 500 & $1.47 \mathrm{E}+02$ & $6.56 \mathrm{E}+05$ & 34.92 \\
\hline$[5-50]$ & 200 & $5.51 \mathrm{E}+01$ & $2.70 \mathrm{E}+04$ & 36.30 \\
\hline [50-80] & 500 & $7.68 \mathrm{E}+01$ & $9.25 \mathrm{E}+02$ & 81.35 \\
\hline [50-80] & 200 & $3.56 \mathrm{E}+01$ & $8.26 \mathrm{E}+02$ & 81.81 \\
\hline [80-125] & 500 & $3.85 \mathrm{E}+01$ & $1.32 \mathrm{E}+02$ & 126.1 \\
\hline [80-125] & 200 & $2.05 \mathrm{E}+01$ & $8.43 \mathrm{E}+00$ & 121.27 \\
\hline$[125-250]$ & 500 & $1.91 \mathrm{E}+01$ & $4.36 \mathrm{E}+01$ & 179.05 \\
\hline$[125-250]$ & 200 & $1.23 \mathrm{E}+01$ & $8.13 \mathrm{E}+00$ & 180.1 \\
\hline
\end{tabular}

Once it has been confirmed that the first-order variability on the beam attenuation is driven by particle concentration [6], one may expect that the discrimination obtained on the first analysis (Fig. 2) could respond mainly to differences in the number of particles at each sample. And therefore, the particle size could have a secondary influence on the spectral shape features. In order to investigate this issue in more detail, spectra with similar total number of particles have been selected and analyzed based on the results estimated by the LISST-100X instrument. In this sense, the cluster analysis has been applied to a subset of spectra with ca. $10^{3}$ particles. The obtained results depicted in Fig. 7 and Fig. 8 show that the spectral shape features associated with particle size are significant enough to be also distinguished on the MDS plot even in a lesser extent. 


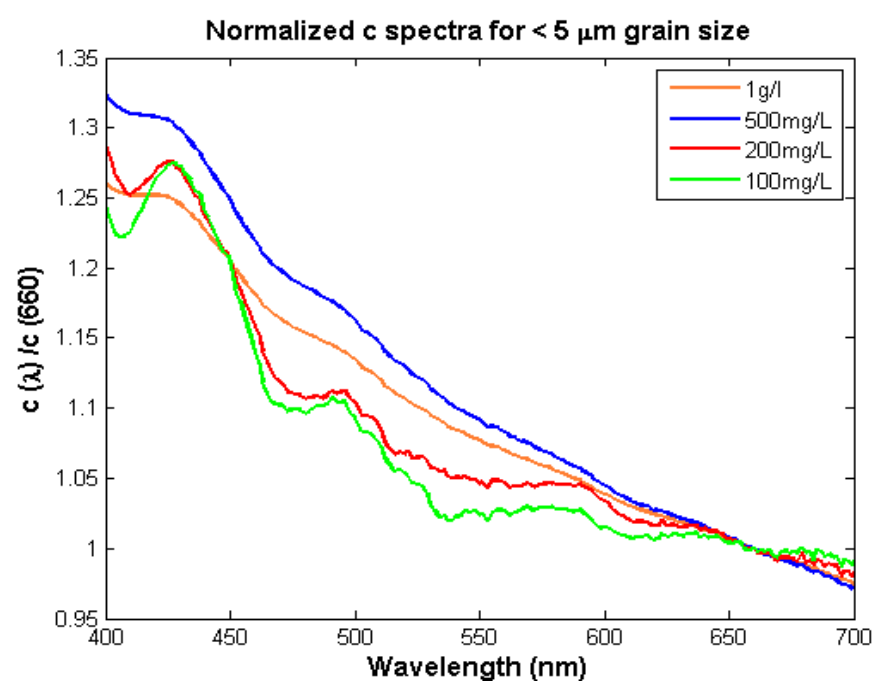

Fig 5. Average normalized beam attenuation spectra for sediment samples with grain size $<5 \mu \mathrm{m}$ at different mass concentrations.

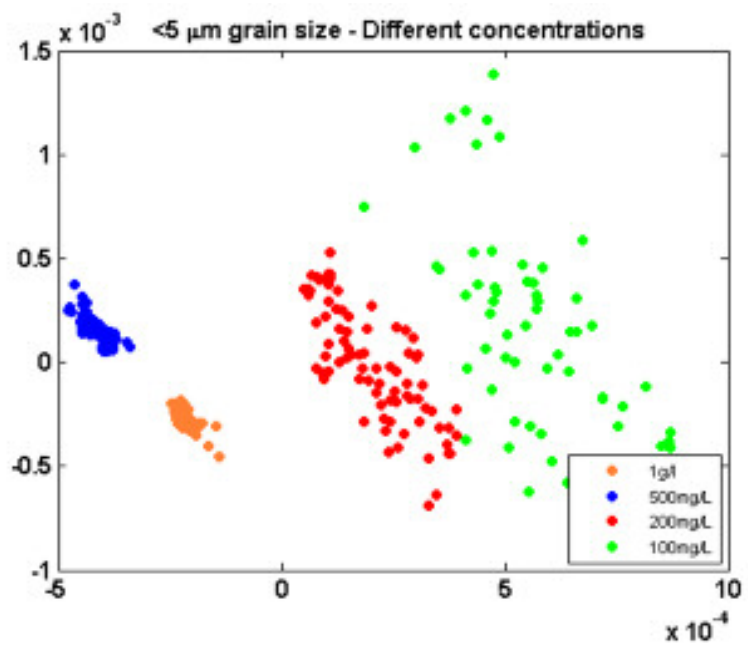

Fig 6. MDS graphic with the classification of the different spectra shown in left panel according to their shape similarities.

With the aim of discriminating specific spectral features related to both particle size and concentration, a deeper statistical analysis has been performed. Nevertheless, our results suggest that the influence of both characteristics on the beam attenuation distributions cannot be easily separated. One possible scenario for the utilization of hyperspectral transmissometers to discriminate sediment samples according to their particle size distribution is to apply our cluster-based approach with a priori knowledge of the mass concentration of the samples.

\section{CONCLUSIONS}

This study investigated the potential of hyperspectral transmissometers on retrieving information about particulate matter characteristics. A VIPER-based assessment was satisfactorily utilized for the characterization of sediment samples in terms of particle size and concentration based on the spectral shape features of the beam attenuation coefficient. Its feasibility for identifying singularities on the beam attenuation spectral shape associated with differences on particle size distribution has been confirmed using an approach based on hierarchical cluster analysis. This method provided a clear and fast classification of samples according to their similarities on the particulate matter characteristics (i.e. particle size and concentration).

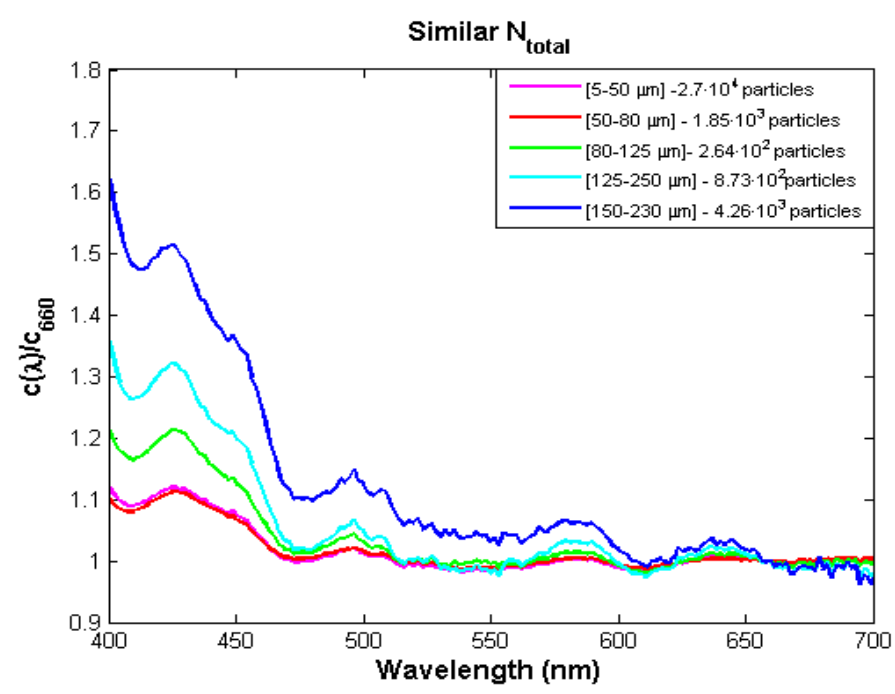

Fig 7. Average normalized beam attenuation spectra for sediment samples with similar particle concentration and different grain sizes.

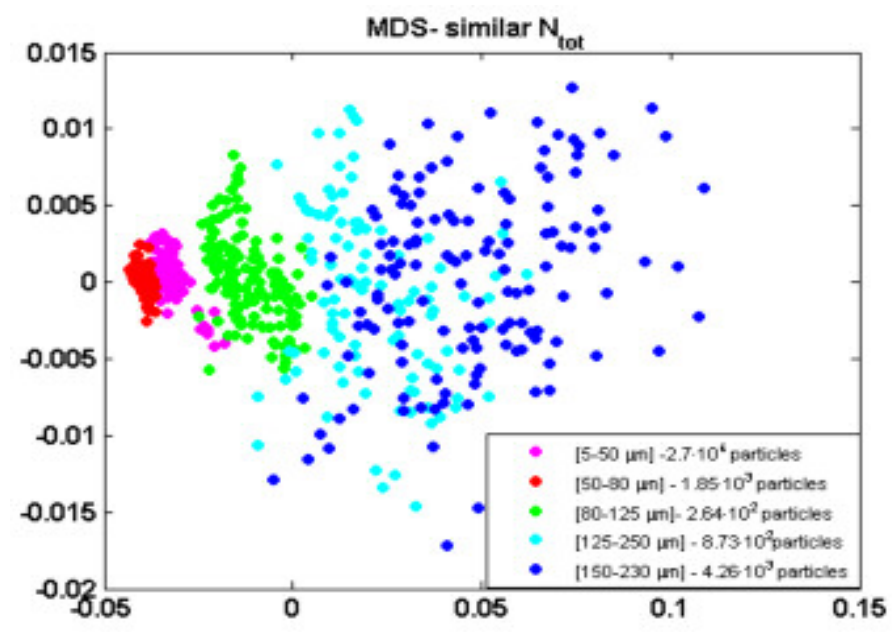

Fig 8. Multi-Dimensional Scaling (MDS) graphic corresponding to samples with similar particle concentration.

For this reason, the use of hyperspectral beam attenuation coefficients as an optical proxy for characterizing the PSD has been proposed as an alternative to other traditional methods such as LISST or Coulter Counter, which are much more expensive and time-consuming approaches.

On the other hand, in order to evaluate the suitability of hyperspectral transmissometers compared to multispectralbased sensors, the same approach was applied to a subset of attenuation spectra at different wavelength resolution. The results showed that the capability of discrimination improves when increasing the spectral resolution. 
Nevertheless, due to the fact that particle concentration drives in first-order the spectral shape features and, hence, overshadows the effect of particle size, a prior knowledge of mass concentration is required in order to be able to discriminate samples with different particle size. Since the experiment yielded promising results, one possible solution would be the combination of VIPER and OBS measurements (as proxy of TSM).

As ongoing work, the proposed methodology will be optimized and applied to field data in order to prove its potential. This method can therefore provide a means for optical oceanographers to better characterize natural complex waters from hyperspectral oceanographic information. Future research will focus on experiments based on hyperspectral data sets of beam attenuation coefficient corresponding to complex estuarine waters. We expect that the evaluation of such data sets will support optimization of the cluster-based approach and other techniques, such as derivative spectroscopy and unmixing techniques, which exploit optical measurements as a source of information on water compounds characteristics.

\section{ACKNOWLEDGMENT}

This study was supported by Total Foundation (PHYTOSCOPE project) and the Spanish Ministry of Economy and Competitiveness (MESTRAL project, reference code: CTM2011-30489-C02-01).

\section{REFERENCES}

[1] Boss E., Pegau W.S., Gardner W.D., Zaneveld J.R.V., Barnard A.H., Twardowski M.S., Chang G.C. and Dickey T.D. (2001). The spectral particulate attenuation and particle size distribution in the bottom boundary layer of a continental shelf. J. Geophys. Res. 106, 9509-9516.

[2] Buonassissi C.J. and Diersen H.M. (2010) A regional comparison of particle size distributions and the power law approximation in oceanic and estuarine surface waters. J.Gephys. Res., VOL 115, C10028, doi: 10.1029/2010JC006256

[3] Reynolds R. A., Stramski D., Wright V. M. and Woźniak S. B.(2010). Measurements and characterization of particle size distributions in coastal waters. Journal of Geophysical Research, VOL. 115 C08024, doi:10.1029/2009JC005930
[4] Diehl P., Haardt H. (1980). Measurement of the spectral attenuation to support biological research in a "plankton tube experiment". Oceanologica Acta- Vol. 3- $\mathrm{N}^{\circ} 1$.

[5] Kitchen J.C and Zaneveld J.R.V (1982). Effect of particle size distribution and chlorophyll content on beam attenuation spectra. Appl. Opt. 21, 3913-3918.

[6] Neukermans G., Loisel H.,Me'riaux X., Astoreca R. and McKee D. (2012). In situ variability of mass-specific beam attenuation and backscattering of marine particles with respect to particle size, density, and composition. Limnol. Oceanogr., 57(1), 2012, 124-144.

[7] Astoreca R., Doxaran D., Ruddick K., Rousseau V., Lancelot C. (2012). Influence of suspended particle concentration, composition and size on the variability of inherent optical properties of the Southern North Sea. Continuental Shelf Research 35 117-128.

[8] Baker, E.T., Lavelle, J.W., (1984). The effect of particle size on the light attenuation coefficient of natural suspensions. Journal of Geophysical Research 89, 8197e8203.

[9] Voss K.J. (1992). A spectral model of the beam attenuation coefficient in the ocean and coastal areas. Limnol. Oceanogr. 37(3), 501-509.

[10] Cheng C., Wei Y., Sun X., Zhou Y. (2012) Estimate Chlorophyll-a Concentration in Turbid Lake Using Spectrum Smoothing and Derivative Analysis. International Journal of Environmental Research and Public Health. ISSN 1660-4601. Doi:10.3390/ijerph90x000x

[11] Mehrubeoglu M., McLauchlab L.L, Zimba P. And Teng M.Y. (2013). Spectral Unmixing of Three-Algae Mixtures Using Hyperspectral Images. IEEE 978-1-4673-4637.

[12] Torrecilla, E., D. Stramski, R.A. Reynolds, E. Millan-Nunez and J. Piera (2011). Cluster analysis of hyperspectral optical data for discriminating phytoplankton pigment assemblages in the open ocean. Remote Sensing Environment, 115, doi: 10.1016/j.rse.2011.05.014:2578-2593.

[13] Butt T., Miles J., Ganderton P., Russell P. (2002).A simple method for calibrating optical backscatter sensors in high concentrations of noncohesive sediments. Marine Geology 192 (2002) 419-424.

[14] Agrawal Y.C., Pottsmith H.C. (2000). Instruments for particle size and settling velocity observations in sediment transport. Marine Geology 168 (2000) 89-114.

[15] Jain, A.K., M.N. Murty and P.J. Flynn (1999). Data clustering: A review. ACM Computing Surveys, 31:264\$323.

[16] Shepard, R.N. (1980). Multidimensional scaling, tree-tting, and clustering. Science, 210:390\$398.

[17] Jonasz M. and Fournier G. (1996). Approximation of the size distribution of marine particles by a sum of log-normal functions, Limnol. Oceanogr., 41, 744-754, doi: 10.4319/lo.1996.41.4.0744 